Ebisu Ebisu

Études japonaises Études japonaises

57 | 2020

Les architectes de l'ère Heisei (1989-2019). Rôles, statuts, pratiques et productions

\title{
JESTY Justin, Art and Engagement in Early Post-War Japan
}

Ithawa (NY), Cornell University Press, 2018, 336 p.

\section{Vincent Manigot}

\section{(2) OpenEdition}

\section{Journals}

Édition électronique

URL : http://journals.openedition.org/ebisu/5456

DOI : $10.4000 /$ ebisu. 5456

ISSN : 2189-1893

Éditeur

Institut français de recherche sur le Japon à la Maison franco-japonaise (UMIFRE 19 MEAE-CNRS)

Édition imprimée

Date de publication : 15 décembre 2020

Pagination : 455-459

ISSN : $1340-3656$

\section{Référence électronique}

Vincent Manigot, « JESTY Justin, Art and Engagement in Early Post-War Japan », Ebisu [En ligne], 57 | 2020, mis en ligne le 20 décembre 2020, consulté le 29 mars 2021. URL : http://

journals.openedition.org/ebisu/5456 ; DOI : https://doi.org/10.4000/ebisu.5456 
(a) Jesty Justin, Art and Engagement in Early Post-War Japan, Ithawa (NY), Cornell University Press, 2018, 336 p.

Enseignant-chercheur à l'université de Chicago, Justin Jesty est spécialiste des rapports entre l'art et les mouvements sociaux de l'après-guerre. Le présent ouvrage se propose d'étudier les liens qui unissent art et politique dans le contexte très particulier du Japon des années 1945 à 1960. Un thème déjà bien traité, mais l'auteur souhaite s'en démarquer en se concentrant sur des pratiques artistiques qu'il qualifie de "démocratiques", et entend montrer que "l'intervention artistique doit dépasser l'idée que l'œuvre d'art ou l'artiste est de manière unilatérale l'auteur de la signification politique» (p. 256).

Pour ce faire, l'ouvrage est divisé en quatre parties thématiques comportant chacune plusieurs chapitres (14 au total). La première ( Arts of Engagement and the Democratic Culture of the Early Postwar ", chap. 1 et 2) s'apparente à une introduction aux théories ensuite développées. Il y est question de ce que l'auteur nomme "culture démocratique ", illustrée par les très nombreux «cercles » ou « groupes » culturels (sākuruサークル) qui se développent alors, en particulier au sein des entreprises. Ce qui rend "démocratiques" ces cercles - et, plus largement, nombre de mouvements artistiques et culturels de l'après-guerre - réside dans la volonté de dépasser les appartenances de classe et le niveau d'éducation de chacun, ainsi que dans le fait que les créateurs conservent les moyens de production comme de diffusion. On peut rapprocher l'importance du collectif, tout comme l'aspect "démocratique " de l'activité artistique, de la nécessité de reconstituer un " ordre social » à une époque où tout est en quelque sorte en ruine (p. 33), mais Jesty souligne que l'art et l'esthétique ont été "considérés comme particuliers par leur capacité à décrire et rendre évidentes les questions politiques et historiques potentiellement abstraites » de l'époque (p. 51).

Pour appuyer son propos, il propose trois études, chacune occupant l'une des trois autres parties du livre. La deuxième partie tout d'abord ("AvantGarde Documentary: Reportage Art of the 1950s ", chap. 3 à 6) traite de l'art du reportage, plus spécifiquement de la "peinture de reportage" (ruporutāju kaiga ルポルタージュ 絵画) et de plusieurs artistes ou 
groupes représentatifs. $\mathrm{Au}$ travers d'événements tels que l'incident du village d'Akebono 曙 ${ }^{1}$ (chap. 3) ou l'incident Matsukawa ${ }^{2}$ (chap. 4), lieux de conflits sur lesquels se rendent ces artistes, l'auteur souligne la faible confiance générale envers les pouvoirs policier et judiciaire ainsi que les médias qui ne traitent pas ou mal ces sujets. C'est surtout la production culturelle (peinture, gravure, photographie, poésie, chanson, films ou encore romans) qui fait connaître ces affaires, aidant parfois à un dénouement plus heureux : ainsi de la conclusion du procès de l'incident Matsukawa, souvent attribuée aux efforts pour le faire connaître (p. 69-70). Jesty fait ensuite intervenir certains des acteurs majeurs de la théorisation des avant-gardes de l'époque - Hanada Kiyoteru 花田清輝, Okamoto Tarō 岡本太郎 ou encore Abe Kōbō 安部公房 - pour tenter de définir ce qu'il nomme « réalisme d'avant-garde» (chap. 5). Enfin, il présente plusieurs voies possibles suivies par les artistes de reportage, illustrées par les exemples de trois d'entre eux (chap. 6). Pour Katsuragawa Hiroshi 桂川寬, le reportage s'intègre dans un projet militant plus large de partage des connaissances qui comprend la mobilisation, l'éducation et la production collective, concrétisé par deux " missions" de groupe de plusieurs mois auprès de poètes ouvriers d'abord, d'habitants d'un village de montagne ensuite. Ikeda Tatsuo 池田龍雄 s'est quant à lui intéressé aux possibilités de la reproduction de masse, notamment par le biais de la presse écrite, la répétition d'une même image permettant d'en démultiplier l'impact. Enfin, indissociable des hésitations et revirements du Parti communiste japonais durant les années $1950^{3}$, Nakamura Hiroshi 中村宏 incarne une conception très différente où les artistes se tiennent désormais éloignés des sites au sein desquels se fabriquaient, justement, les reportages, mais aussi de l'échange que les rencontres faisaient naître.

Comme l'annonce son titre, la troisième partie («Opening Open Doors: Sōbi and Hani Susumu ", chap. 7 à 10) offre d'abord (chap. 7 à 9) une étude portant sur l'Association pour une éducation à l'esthétique créative (Sōzō biiku kyōkai 創造美育協会 ou Sōbi 創美) et deux de ses personnages-clefs : son fondateur Kubo Sadajirō 久保貞次郎 et le peintre Kitagawa Tamiji 北川民次, qui en fut membre avant de s'en détourner. Celle-ci a pour objectif de mettre l'éducation artistique au cœur de la formation des enfants, la considérant non comme une matière mais " comme le modèle de tout apprentissage " (p. 130). L'éducation y est en outre conçue comme exclusivement 
non-autoritaire, enseignant et apprenant y étant sur un pied d'égalité. À une époque où l'éducation est décentralisée, cette association est populaire auprès des enseignants (souvent inquiets du rôle que l'école a pu jouer pendant la guerre) qui peuvent échanger au travers de groupes d'étude locaux. Mais son influence devait diminuer en même temps que leurs marges de manœuvre avec les réformes de l'éducation de la fin des années 1950. Enfin, le chapitre 10 se concentre sur trois ouvres du cinéaste Hani Susumu 羽仁進, parmi lesquelles $E$ o kaku kodomotachi 絵を書く子供たち (Les enfants qui dessinent, 1956), tourné dans la classe d'un jeune professeur affilié à Sōbi. Plus généralement, Jesty considère que la proximité entre Hani et Sōbi tient à la manière dont "sa caméra et le film étaient "du côté de l'enfant" à peu près de la même manière que l'enseignant de Sōbi » (p. 167).

La quatrième partie ( Kyushu-ha Tartare: Anti-Art between Raw and Haute ", chap. 11 à 14) traite quant à elle du groupe Kyūshū-ha 九州派 (École de Kyūshū), représentatif de l'anti-art et dont les membres, originaires de l'île du même nom et donc très éloignés de la capitale, n'ont pour la plupart guère de formation artistique. S'illustrant dans la peinture, la sculpture ou la performance, beaucoup utilisent des matériaux alors inhabituels, avec une prédilection pour le goudron. Le groupe se différencie de l'art du reportage autant par la manière d'envisager la " démocratisation " de l'art que par le profil des artistes qui le composent : il ne s'agit plus ici d'apporter l'art où il est peu présent, mais de partir du peuple pour y rester, les membres de Kyūshū-ha étant eux-mêmes issus des classes populaires. Se voulant accessible à tous, "acteurs" (n'importe qui peut devenir membre) comme "spectateurs» (plusieurs expositions dans la rue), le groupe ne rencontre cependant pas le succès, peut-être du fait du caractère grotesque voire scandaleux, et donc paradoxalement très peu accessible, de ses œuvres (p. 215). Le chapitre 13 présente quatre des membres de Kyūshū-ha, Sakurai Takami 桜井孝身, Tabe Mitsuko 田部 光子, Kikuhata Mokuma 菊畑茂久馬 et Miyazaki Junnosuke 宮崎準之助, illustrant ainsi une autre caractéristique du groupe : son hétérogénéité. Enfin, le chapitre 14 se penche sur l'art contemporain japonais et le rôle que la presse a joué dans la définition et la mise en place de celui-ci.

Quelques remarques. La question de l'éducation artistique des enfants, abordée dans la troisième partie, est passionnante. Au lendemain de la guerre, nombre de mouvements artistiques prônant la liberté créatrice et/ ou l'éducation artistique ont vu le 
jour; ainsi de Sōbi. Mais quelques précisions supplémentaires auraient été souhaitables. L'auteur donne à plusieurs reprises l'impression que les expérimentations artistiques de l'après-guerre n'ont point connu de précédent avant ou pendant le conflit. S'il existe indéniablement un avant et un après 1945, la question de la rupture est souvent à nuancer et celle de la continuité tout aussi importante. Ainsi, le nom de Yamamoto Kanae 山本鼎 (1882-1946) n’apparaît que dans une note discrète (n. 4 p. 288). Peintre et graveur, il est pourtant un précurseur de l'éducation artistique des enfants : il prononce en 1918 une conférence en forme d'appel au dessin libre des enfants, organise des expositions dès l'année suivante, et publie en 1920 Jiyūga kyōiku 自由画教育 (L'éducation au dessin libre) dans lequel il précise sa pensée, proche de celle de Kubo ${ }^{4}$. Contemporaines de Sōbi et, là encore, à rapprocher, on aurait également pu souhaiter qu'il soit question des expérimentations de Gutai 具体 (seulement mentionnées).

Par ailleurs, Art and Engagement est un ouvrage qui demande au lecteur un certain bagage culturel. Il est par exemple fait référence dans le dernier chapitre du livre à "Neo Dada» (p. 248 et 250-251), sans préciser ce dont il s'agit, et il n'est pas évident de comprendre qu'il est question non pas du travail d'artistes américains des années 1950-1960 (Robert Rauschenberg ou Jasper Johns), mais d'un très éphémère groupe fondé à Tokyo en 1960, entre autres par Arakawa Shūsaku 荒川修作 et Akasegawa Genpei 赤瀬川原平 (certes cités par Jesty), et plutôt d'ailleurs (dans un premier temps du moins) nommé $\mathrm{Neo}$ Dadaism Organizers 5 . De même, l'auteur évoque certains personnages-clefs, sans toujours les présenter : ainsi de Hariu Ichirō 針生一郎 et Hanada Kiyoteru, seulement qualifiés de "critique " pour le premier (p. 50, 169, 198, 235, etc.), de " penseur " pour le second (p. 91).

En fait, ces remarques en appellent une autre, plus générale: malgré ce qu'annonce Jesty en introduction (p. 5), l'ouvrage traite finalement assez peu de politique - ou le fait en filigrane -, et les conclusions qu'il tire sont parfois difficiles à saisir pour un non-spécialiste. Pareillement, beaucoup d'œuvres sont évoquées mais très peu sont étudiées en détail. Même courtes, davantage d'analyses et des contextualisations relatives à la politique comme à l'art auraient rendu la lecture plus facile tout en donnant plus de poids aux arguments, souvent pertinents.

Ces quelques remarques n'enlèvent cependant rien à la très grande qualité du livre, et c'est même ce qui les motive: Art and Engagement est 
un ouvrage assez exigeant qui se lit comme une histoire - c'est une qualité -, souvent passionnante, riche en découvertes, et donne envie d'en savoir davantage. Nombre d'œuvres sont en outre présentées dans le corps du texte, auxquelles s'ajoutent deux cahiers d'illustrations couleurs. Jesty convoque de nombreuses sources premières et offre un travail dense et sérieux sur un pan de l'art japonais peu traité, notamment en langue occidentale. La peinture de reportage, tout particulièrement, bénéficie ainsi d'un éclairage bienvenu. Une excellente lecture.

\author{
Vincent Manigot \\ Professeur assistant \\ à l'université Waseda
}

après la réunification de 1955 , ce qui sonne pour beaucoup comme une trahison et un énième avatar de «réorientation" (tenkō 転向).

4. Voir "Jiyūga kyōiku no yōten" 自由 画教育の要点 (Principes du dessin libre), in Yamamoto Kanae, Jiyüga kyöiku, Tokyo, Ars, $1921: 1-23$.

5. En langue occidentale, voir Lucken Michael, L'art du Japon au vingtième siècle, Paris, Hermann, 2001 : 193-194; Chong Doryun, Hayashi Michio, Sumitomo Fumihiko \& Kajiya Kenji, From Postwar to Postmodern, Art in Japan, 1945-1989Primary Documents, New York, Museum of Modern Art, $2012: 160$.

1. Le village d'Akebono est situé dans le département de Yamanashi. L'incident en question (Akebono jiken 曙事件) s'y déroula en 1952, lorsqu'un groupe de villageois séquestra et blessa un riche propriétaire terrien et plusieurs de ses proches pour protester contre celui-ci.

2. L'incident Matsukawa (Matsukawa jiken 松川事件) est le déraillement d'un train en 1949 dans le département de Fukushima, qui entraîna la mort de trois agents qui se trouvaient à bord. Vingt personnes travaillant pour les chemins de fers nationaux ainsi qu'à l'usine Toshiba locale, toutes syndiquées, furent arrêtées et accusées de sabotage, puis finalement innocentées en 1963.

3. Scission en 1950 , puis adoption d'une stratégie de lutte armée qui sera critiquée 